\title{
Revolutionizing science through simulation: A junior researcher's perspective on research challenges in uncertain times
}

\author{
Baskar Ganapathysubramanian, Assistant Professor, Mechanical \\ Engineering, Iowa State University
}

S imulation science can serve as a powerful strategy to explore and understand complex phenomena. It is especially suited to gaining insights into events occurring at extreme scales (very small and fast or large and slow), rare events (hurricanes, earthquakes), or events that involve interplay of a large number of phenomena (biological processes, multi-scale phenomena). Progress in cyber infrastructure (hardware) and concurrent development in computational tools (software) place practitioners of simulation science in a unique position. They can serve as a bridge between experiments and theory, supplement experimental observation with computational insight, and enable high throughput exploration and design. I show specific examples of how simulation science can effectively bridge disparate disciplines to solve complex problems. Such multidisciplinary activities seem to increase the possibility of research funding. Based on these experiences, I share my opinions on how administrators can enable faculty to successful multidisciplinary teams.

My research group has domain expertise in developing high performance computational methods and associated tools for modeling and controlling transport phenomena, particularly in the context of advanced energy generation and storage. This is an emerging topic of research owing to the ever-increasing need for high-performance materials, devices, and processes in sustainable energy applications. We work on integrating three key scientific issues related with this effort, namely: (1) accounting for the multi-scale, multi-physics nature of these processes, (2) the necessity of accounting for the inherent uncertainty in these processes, and (3) the importance of experimental validation and verification of any modeling framework. This scientific viewpoint has encouraged us to work very closely with experimental scientists and practitioners to ensure that our computational advances directly enable the science problem at hand. This has resulted in very productive partnerships with a variety of research groups (architects, control scientists, computer scientists, civil engineers, agronomists, chemists, physicists and mathematicians). I illustrate a few concrete examples of this collaborative viewpoint.

Simulation science for sustainable buildings:

The construction, operation, and maintenance of buildings require an enormous amount of energy. Buildings consume approximately $36 \%-41 \%$ of the U.S. total annual energy usage and about 
$40 \%$ of the global energy consumption [13]. Even Heating Ventilation and Air Conditioning (HVAC) systems alone for the U.S. use approximately $16 \%$ of the U.S. annual energy usage [3]. In recent years, there has been a conscientious push towards developing sustainable methods of designing buildings. Designs that leverage natural flows (wind and buoyant ventilation), and passive heating/cooling (thermal mass effects) hold promise for energy efficiency. To efficiently leverage these naturally available energy flows (passive solar heating, natural ventilation and cooling strategies), various elements need to operate together -- windows need to be opened or closed, shading devices opened or closed. Additionally, active systems need to be activated to start operation once the natural forces are no longer available at proper strength.

This vision calls for a holistic approach towards the design of space, envelope, and environmental control and operation systems in order to reduce their impact on energy resources and the environment. Motivated by this, we formed an interdisciplinary team consisting of an architect, a computational scientist, a control theorist, and a material scientist. We are working on coupling engineering expertise in computational fluid dynamics (CFD), advanced control system design with architectural design and human factors to build simulation expertise and associated tools for designing free ventilation, heating and cooling strategies in green buildings.

The overarching challenge with integrating sustainable strategies for conditioning the indoor space is the ability of sustainable buildings to consistently maintain indoor occupant comfort. That is, under a variety of external conditions, the building design should ensure a reasonable modicum of comfort for the occupants. While this task is inherently difficult, there is historical precedent of sustainable buildings that achieve reasonable indoor comfort under extreme outdoor weather conditions. A particularly relevant set of architectures include domed houses in Turkey and Syria, and wind-catchers in Iran. With this motivation, we have been studying the adobe houses in Harran, Turkey. This is part of our long-term goal of comprehensively understanding, and incorporating promising features of the Harran house (channeled natural ventilation co-opted with thermal mass effects) into sustainable designs in the US and Turkey. These vernacular buildings constructed with mud and bricks provide agreeable thermal comfort levels even under very high ambient temperatures, high solar radiation, and low precipitation levels. Thermal comfort is maintained via an interesting interplay between thermal mass effects and natural ventilation.

We use simulations as a powerful tool to help predict flow physics and identify problematic regions of existing buildings, and aid in the design and optimization of energy efficient newly constructed buildings. Computational Fluid Dynamics (CFD) simulations break up the building into a set of pieces, also known as mesh elements, and resolve the governing equations of fluid flow and the energy equation on each piece/element. This discretization process of the building zone allows CFD simulations to eas- 
ily analyze thermal comfort in many different places in the occupied zone and identify ventilation patterns. This provides a detailed picture of how energy and air flow in the building. We have made progress in developing mathematical formulations for modeling natural ventilation in complex geometries. Note that it is difficult to model natural ventilation as it constantly fluctuates in real time in response to changes in wind direction and intensity [4]. Standard computational fluid dynamics approaches (CFD) based on Reynolds-Averaged-Navier-Stokes (RANS) models have been shown not to work for this class of problems. Recent results suggest that a more high-fidelity approach using LargeEddy-Simulation approach would enable accounting for the effects of natural ventilation [5]. This calls for a concerted high-performance computing based approach to design, explore and benchmark the ability of LES to account for natural ventilation. We have very promising preliminary work on a massively-parallel finite element method variational multiscale based LES framework for natural ventilation.
This collaborative effort has enabled us to integrate architecture with high performance computing towards understanding how thermal mass effects and natural ventilation affect comfort in the Harran house. We are able to unravel the competing effects of buoyancy and wind driven natural ventilation, thermal mass, short-wave and long-wave radiation, conduction, convection, wind thermal energy, and surface roughness. We have shown that this model performs very well in comparison with experimental measurements made at the Harran house. This sets the stage for future developments in adaptive controls and architectural design to extend these concepts to sustainable buildings in the US.

Simulation science for sustainable solar energy:

Organic solar cells (OSC) represent a very promising low-cost, rapidly deployable strategy for harnessing solar energy. Organic solar cells manufactured from organic blends are amenable to roll-toroll processing on flexible substrates, have high optical absorption coefficients, allow low-temperature processing, and

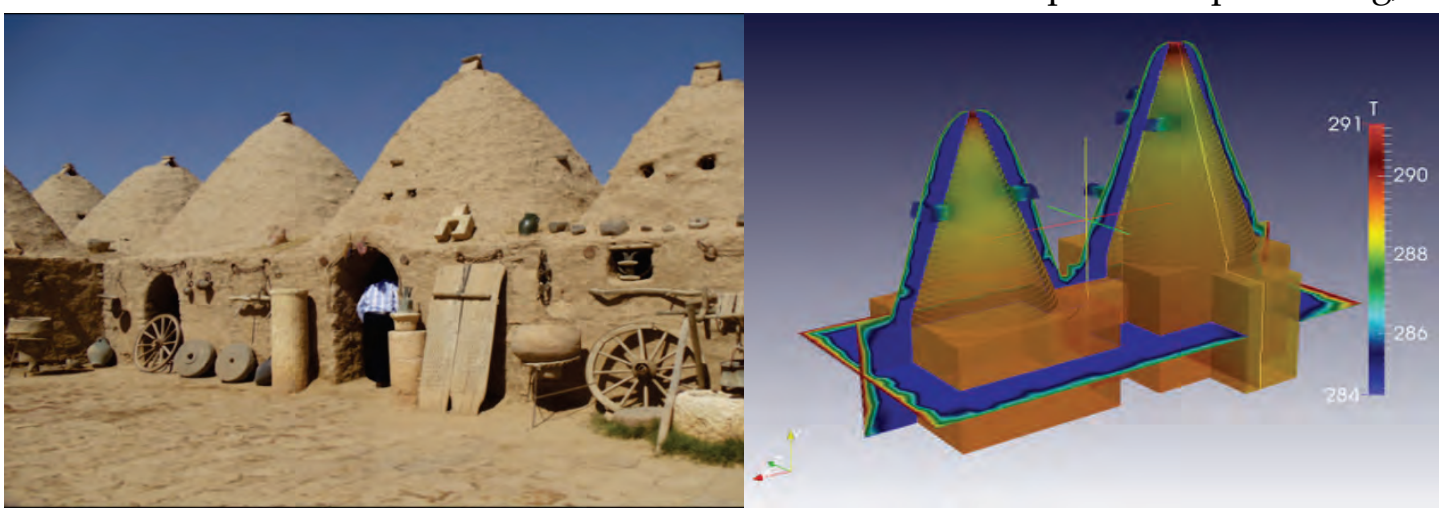

Figure 1: Left: Picture of the Harran house. Notice the set of domed, corbelled roofs. Right: A high resolution computational fluid dynamics analysis of the house. This reveals that the thermal mass of the thick walls and the natural convection play equally key roles in maintaining reasonable temperatures indoors. 
are easily tunable by chemical doping. The past decade has witnessed considerable advances in organic photovoltaic technology, both in terms of understanding of physical aspects of the underlying processes and in improvements in power conversion (PCE) efficiencies (from PCE below $3 \%$ to currently the highest reported efficiency $9 \%$ obtained in laboratory conditions). This improvement was made possible through a three-pronged approach: (i) new materials development, (ii) new device designs, and (iii) morphology control via processing.

Despite these significant advances, wide-spread use and profitable commercial production of OSCs are currently limited. These goals await an increase in PCE to the $15-20 \%$ range and an improved life span. Two key advances are necessary to improve PCE. The first is a predictive framework enabling practitioners (chemists, material scientists, and manufacturing) to obtain targeted morphologies by tailoring competing phenomena during manufacturing. Secondly, we must be able to quantify the relationship between morphology and photovoltaic performance. Taken together, the need for these advances presents a fundamental materials barrier to understanding the processstructure-property relationship governing OSC behavior. These challenges -and moving beyond experimentally dominated, trial and error strategies -serve as compelling reasons to develop computational frameworks that can be used as virtual manufacturing tools and virtual characterization tools for detailed, rapid and cost-effective analysis and design of organic solar cells. My group is developing computational tools in conjunction with experimental groups at KAUST (Saudi Arabia), NIST, UC Davis, UC Santa Barbara, Academica Sinica (Taiwan), and Imperial College (England). Some integrative activities include:

A new paradigm for computational science and predictive modeling in organic photovoltaic technology: This collaborative group has formulated, implemented and verified a computational framework of morphology evolution during solventbased fabrication that approaches device scale. This work is of significance, taking into account that this field is an experimentally dominated field and faces significant challenges related to morphology visualization, and linking processing conditions with morphology. This research offers unique possibilities to explicitly visualize the temporal morphology evolution of 3D structure, including internal structure, from early stages to the final structure. Our work allows predicting the three-dimensional nano-morphology evolution over large time and space scales during solvent-based fabrication of thin film organic electronics, which has enabled a deeper understanding of the physics involved in this process and thus, allows the design of better electronics.

A major hindrance to the efficient and commercial production of organic solar cells is the optimal choice of fabricating conditions out of innumerable possibilities. These include choosing from a wide variety of solvents and mixtures of solvents, the substrates used for fabrication, the applied fluid stresses, and the temperature at which fabrication and post-treatment annealing is performed. Experimentally exploring these infinite 
possibilities is infeasible. Our recent work has resulted in virtual frameworks that model the fabrication of organic photovoltaic cells. This is analogous to the development of virtual wind tunnels that accelerated the design and development of the next generation of aerodynamic cars and airplanes.

In conjunction with experimental colleagues, we have developed methods of characterizing morphology using a graph-based approach. While earlier analysis of imaged structures was purely visual and thus only qualitative, the graph-based approach brings a greater degree of quantitative rigor to this aspect of the field. The analysis facilitates the prediction and interpretation of time-varying three-dimensional snapshots of intermediate morphologies and enables high-throughput sorting of morphologies. The high throughput sorting has provided valuable insights towards more targeted synthesis and materials design.
It has also enabled the selection of optimum blend compositions; and, thus, significantly reduced the design time-line for next-generation organic photovoltaic devices. This tight integration of experimental and computational efforts has resulted in substantial breakthroughs in this field.

Simulation science for engineered plants:

Crop production has to double over the next decade to support population growth and altered eating habits (e.g., increased animal production in Asia). This has to be accomplished on the same or even a reduced area of available land. At the same time, climate change may lead to increasingly adverse environmental conditions for crop production. Recent events such as the extreme drought of 2012 showcase the urgency and need for producing more resilient crops. Current methods to engineer crop performance are based on expensive, agronomic experiments in multiple field environments

Figure 2: (A) Comparison between our numerical results and experimental results, $(B)$ Difference in morphology evolution due to two different solvents. The arrow points along increasing time and decreasing height of the film layer [62]. (C) Representative 3-D APFO3:PCBM nano-morphology. The two slices below show the effect of two different evaporation rates on the average feature size of the morphology.

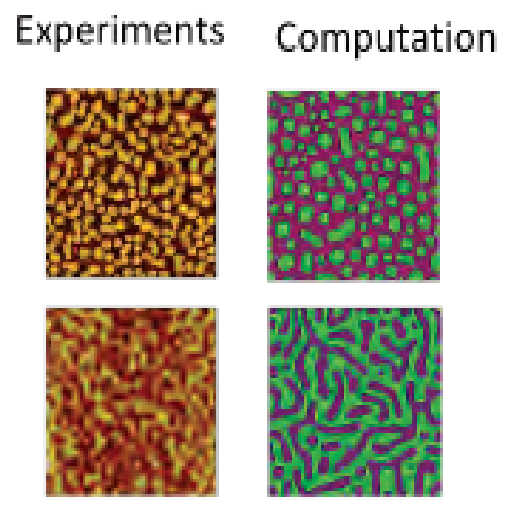

(A)

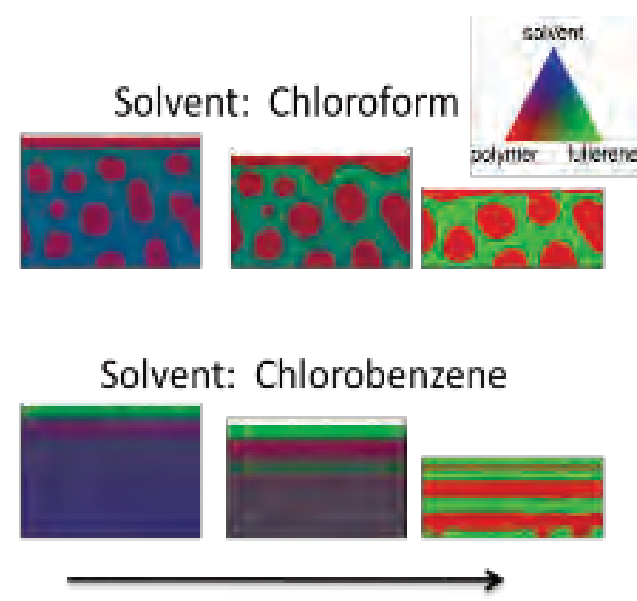

(B)

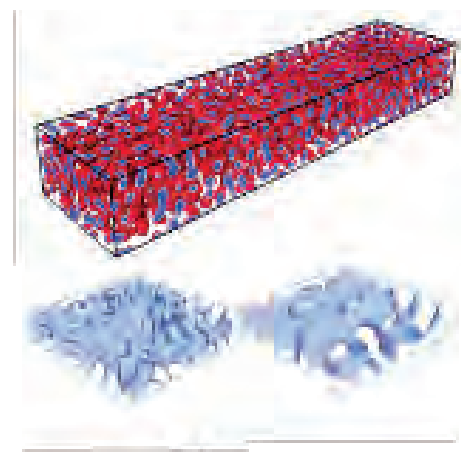

(C) 
that link genotypes and phenotypes with crop performance. There is, thus, a need for establishing reliable associations between DNA markers, phenotypes and crop performance, before DNA markers can be directly used for engineering crop performance. My group is part of a working group (consisting of agronomists, engineers, statisticians and computational scientists) that built on the hypothesis that an integrated approach combining genetic experiments (genomics and highthroughput phenotyping), advanced phenomics (high resolution $x$-ray visualization, lab-on-a-chip measurements), physics-based numerical modeling (nutrient and water transport and uptake, root growth dynamics) will deliver reliable, predictive and cost-effective associations between genotypes, phenotypes, and crop performance. A specific focus has been to use computational fluid mechanics and mathematics to describe how the phenotype of plants controls tolerance to drought. These models will enable us to discover plant phenotypes (i.e. traits) with optimum resistance to drought.

\section{How can institutions help:}

These three examples provide concrete evidence that a multi-disciplinary approach involving experimental and computational expertise can be leveraged to solve complex societal problems (which could ensure a steady source of research funding). These examples also suggest that institutions can enable and proactively encourage such research teams. These activities can be divided into three classes: Initiate, Encourage, and Enable. I briefly outline how institutions can do (and are doing) so:
Encourage: Universities must encourage faculty to aspire tow ards a diversified portfolio of problems as well as funding agencies to target. There has to be a conscious move away from a 'goldstandard' of funding from a single federal source (like NSF in engineering, or NIH in medical sciences). This includes a healthy distribution of funding between industry and federal sources. One way to accomplish this is to actively encourage faculty to have a healthy distribution of short term and long term research projects, as well as to inculcate interest in collaborative efforts: multi-, inter- disciplinary. Fundamental research integrated with applied research (associated with targeted applications) is easier to articulate and argue for, especially with mission specific agencies (DoD, DARPA, DOE, etc.). Iowa State University encourages faculty to do this by providing a clear message that team oriented large projects are valued.

In addition, there has to be clear feedback and reward/advancement for faculty following this strategy. Promotion and tenure documents may look different in this context of multi-disciplinary work, with half-dozen or more co-authors on papers, jointly mentored students, and multiple co-PI's on grants becoming the norm. The administration should have clear guidelines for faculty to articulate their contributions for P\&T as well as awards/recognition. While a focus on foundational research is important, departments should not discourage junior faculty from participating in large grants.

Initiate: The university and college can initiate research in strategic areas that 
are of relevance at the university/state/national level. For instance, ISU recently launched the Presidential Initiative for Interdisciplinary Research "to support research efforts that could lead to major advances, discoveries and technologies". These are awards in areas that are (a) of strategic importance to ISU, (b) core strengths to ISU, or (c) topical with a high return on investment. These awards enable the formation of large teams and provide pursuit funding. In addition, colleges have similar (albeit smaller scale) programs that initiate activities of strategic interest at that scale. This has multiple ramifications - as an internal funding mechanism in a funding climate where insistence on preliminary data is widening, as an incubator of entrepreneurial ideas, and for providing successful role models for junior faculty to aspire towards.

Enable: Universities should play a key role as an enabler by providing trained support staff who are well versed in the budgetary and regulatory intricacies of various funding sources (NSF vs $\mathrm{NIH}$ vs DoD). Universities should also enable faculty to nurture relationships. An example of this is providing travel grants to visit funding managers across the country. This is clearly a low-risk, high-reward investment for the university. Additionally, universities can try to make industrial partners feel welcome by making IP issues straightforward. Universities can also streamline activities and enable formation of teams by making strategic investments, and laying down guidelines for internal competitions for limited submission proposals. A case in point is the NSF MRI call, which results in a feeding frenzy of a diverse set of internal proposals every year. ISU has identified and disseminated a multi-year list of strategic ideas for this competition (Year 1: HPC, Year 2: materials, Year 3: Bio etc.). This has significantly bolstered team building and has encouraged various groups to work together to form teams with a concrete multi-year plan. The multi-year list also serves as a template for making strategic hires.

Universities (especially in the Midwest) can leverage existing facilities to create win-win condition by actively collaborating to enable large scale centers. This avoids duplication of infrastructure in a narrow geographic area and can enable significant cost-matching. Buy in from the faculty can be cemented by making other university faculty part of centers, and by awarding courtesy appointments. It appears that this is strategically promising for the group of universities attending the Merrill conference in the areas of engineering sciences, agriculture, and medicine.

Acknowledgements: I thank Mabel Rice for her kind invitation to be a part of the 2014 Merrill Conference and Evelyn Haaheim for making the experience so enjoyable. Several of these opinions are a result of extensive discussions with Sriram Sundararajan and Balaji Narasimhan at ISU. I am thankful to them for taking time to talk to me about these issues.

References

[1]EPA, http://www.epa.gov/oaintrnt/projects/, 09/10/2013.

[2] United Nations Environment Programme, www.unep.org/sbci/AboutSBCI/Background.asp, 2013. 
[3] Department of Energy,"Buildings Energy Databook,"

[4] Allard, Francis Santamouris M., and Alvarez Servando. Natural Ventilation in Buildings: A Design Handbook [in English]. London: James and James (Science
Publishers) Ltd, 1998. Government publication (gpb); International government

publication (igp); CD for computer (cdc). 